The Astrophysical Journal, 456:L127-L130, 1996 January 10

(C) 1996. The American Astronomical Society. All rights reserved. Printed in U.S.A.

\title{
AN ALTERNATIVE EXPLANATION FOR THE OBSERVED VARIATION OF SOLAR $P$-MODES WITH THE SOLAR CYCLE
}

\author{
THOMAS JOSEPH LYDON ${ }^{1}$ \\ Center for Solar and Space Research, P.O. Box 208101, New Haven, CT 06520-8101 \\ D. B. GUENTHER \\ Department of Astronomy and Physics, Saint Mary's University, Halifax, NS B3H 3C3, Canada \\ AND \\ S. SOFIA \\ Center for Solar and Space Research, P.O. Box 208101, New Haven, CT 06520-8101 \\ Received 1995 May 30; accepted 1995 September 27
}

\begin{abstract}
By mimicking the effects of a magnetic field on the interior structure of a solar model, observed changes in $p$-mode frequencies over the course of the solar cycle are explained in terms of a change in the intensity and distribution of a magnetic field near the top of the solar convection zone; previous attempts of explain these observations have concentrated on magnetic fields extending into the solar atmosphere. Specifically, the observed frequency changes for the $5 \leq l \leq 60$ modes between 1986 and 1989 can be accounted for by a change in magnetic field strength of $400 \mathrm{G}$ approximately $320 \mathrm{~km}$ below the solar surface; in a standard solar model, this depth corresponds to a temperature of $10,800 \mathrm{~K}$ and a pressure of $3.63 \times 10^{5} \mathrm{dyn} \mathrm{cm}^{-2}$. These results are discussed in light of a measured change in the solar radius of $4 \mathrm{~km}$ between 1992 and 1994 by the Solar Disk Sextant Experiment.
\end{abstract}

Subject headings: Sun: interior — Sun: magnetic fields — Sun: oscillations

\section{INTRODUCTION}

Libbrecht \& Woodard (1990) have measured changes in the solar $p$-mode frequencies between 1986 and 1989, and an understanding of the physical processes responsible for such changes could provide a important clue to the inner workings of the solar cycle. Goldreich et al. (1991) have demonstrated how these observed changes could be produced by a magnetic field extending from the solar atmosphere to deep within the solar interior. The intensity of their proposed field ranged from $30 \mathrm{G}$ (exterior) to $2000 \mathrm{G}$ (interior) with an intensity of roughly $250 \mathrm{G}$ at the solar photosphere. A similar effort by Jain \& Roberts $(1993,1994)$ demonstrated that the observed frequency changes could be explained as well by a change in the chromospheric magnetic field $(\Delta B \sim 10 \mathrm{G})$ and a simultaneous change in chromospheric temperature $(\Delta T \sim 1000 \mathrm{~K})$. Our work shares some aspects of these two previous efforts. As in Goldreich et al. (1991), the effects of a magnetic field have been parameterized in terms of a change in magnetic pressure and entropy. In addition, Goldreich et al. (1991) concluded that a horizontal magnetic field would lower $p$-mode frequencies, and we have found a similar result. As with Jain \& Roberts, we have included the effects of the solar convection zone on the $p$-modes by modeling the entire sun from atmosphere to core. Despite similarities with earlier efforts, we contend that the observed increase in $p$-mode frequencies between 1986 and 1989 can be explained by a decrease in magnetic field intensity within a narrow region of the solar interior. This proposed field is horizontal in nature and is therefore distinct from the vertical fields at the solar surface, which showed an increase in intensity over the same period. While this hypothesis is not without its own difficulties, the

${ }^{1}$ Global Change Distinguished Postdoctoral Fellow, US Department of Energy, Office of Health and Environmental Research. explanation requires neither an intense field in the solar atmosphere (Kuhn 1995 and Lin 1995 contend that such fields are not observed) nor a large change in chromospheric temperature. The intention, however, is not to criticize other efforts, but rather to suggest an alternative mechanism for $p$-mode changes which may also be able to explain consistently solar radius and luminosity changes over the course of the solar cycle.

\section{TECHNIQUE}

Our method for calculating the effects of magnetic fields on $p$-modes relied upon three elements: a solar model, a means of modifying the structure of the solar model for the field, and a means of calculating the frequencies. The basis for the solar model was the Yale Rotating Evolution Code (Guenther et al. 1992). The numerical accuracy of the code was improved to the point that the evolutionary changes experienced by the Sun over the course of a year could be resolved fully. The effects of a magnetic field were treated as a pressure and internal energy perturbation on the structure of the model. This technique was developed to encourage the use of stellar structure codes for the study of stellar variability and was a revision of an earlier effort by Endal, Sofia, \& Twigg (1985). An important result from this work was that the effects of even a very localized magnetic field can extend throughout the model rather than being confined to the perturbed region (Lydon \& Sofia 1995). The third component was Guenther's nonradial, nonadiabatic, stellar pulsation program (Guenther 1994).

\section{FIRST ATTEMPT TO REPRODUCE CHANGES}

Figure 1 compares the observed frequency changes for the modes $5 \leq l \leq 60$ (binned and averaged) with the calculated non-adiabatic changes for the $l=0,20,30,40,50$, and 60 


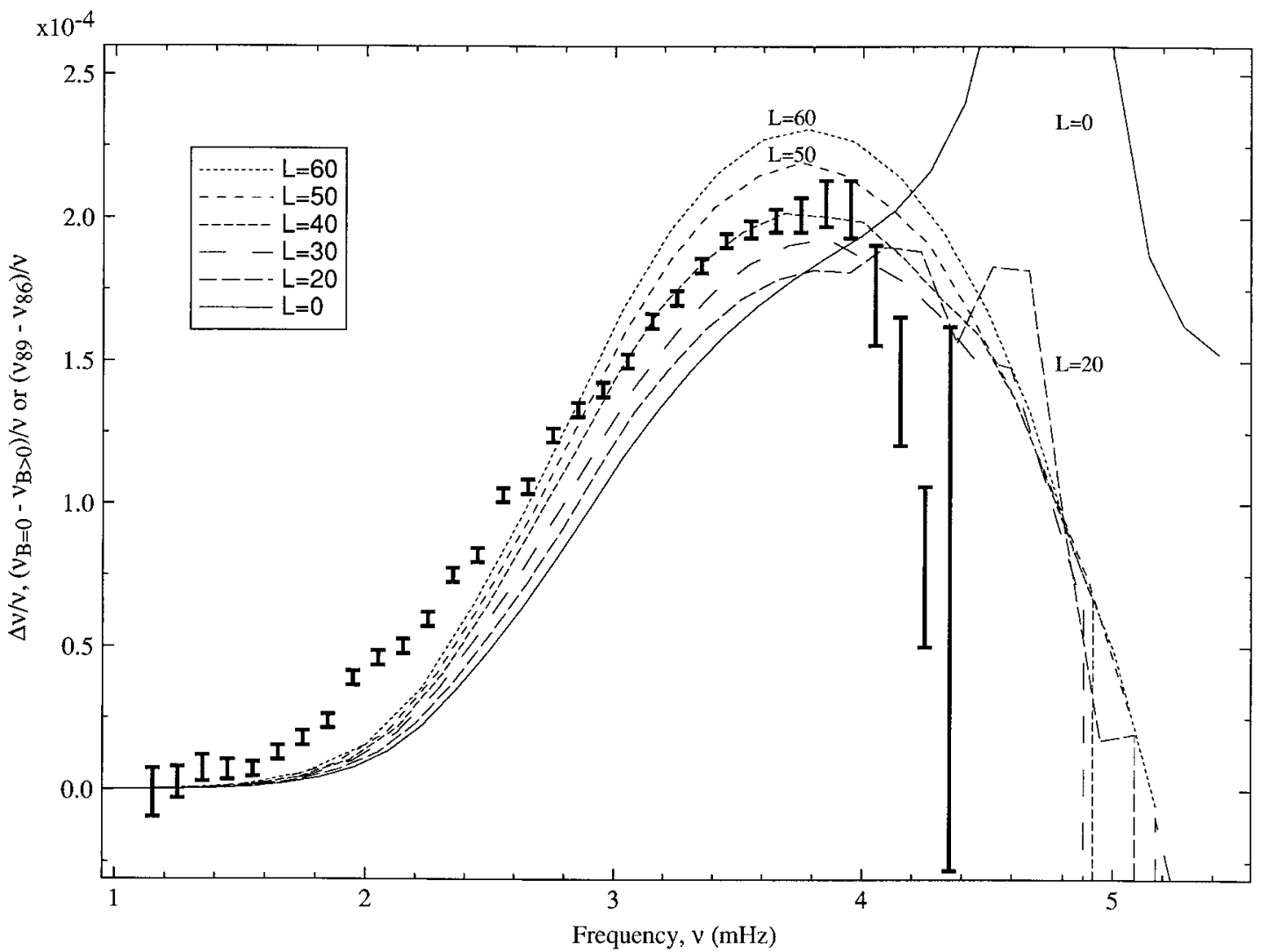

FIG. 1.-A comparison of observed and calculated frequency differences. For the calculated frequencies, note (1) the small trend of larger differences for higher $l$-modes near $3800 \mu \mathrm{Hz},(2)$ the similarity of all modes at low frequencies, and (3) the erratic behavior near $5000 \mu \mathrm{Hz}$.

modes. The introduction of a magnetic field with radial dependence but no radial direction (locally, such a field would be horizontal) lowered all frequencies less than $4800 \mu \mathrm{Hz}$. The field was the result of a "magnetic perturbation" of a thin shell in the outer layers of a solar model. While a spherically symmetric field is not a solution of Maxwell's equations, the symmetry of the solar model required this gross approximation. The maximum intensity of the field was $400 \mathrm{G}$; the field was located $320 \mathrm{~km}$ below the solar surface, a depth corresponding to a temperature of $10,800 \mathrm{~K}$ and a pressure of $3.63 \times 10^{5}$ dyn $^{-2}$. Employing the variable $M_{D}$ defined as

$$
M_{D}=\log \left[1.0-\left(M_{r} / M_{\odot}\right)\right],
$$

where $M_{r}$ is the mass contained with radius $r$ and $M_{\odot}$ is the solar mass, the field was located at $M_{D}=-9.5$. The distribution of the field was Gaussian with respect to the variable $M_{D}$ with $\sigma=0.25$, which corresponded to an FWHM of approximately two local pressure scale heights.

With regard to the calculated differences in Figure 1, several features are worth pointing out. While there was only a slight dependence of the frequency change on $l$, a trend was obvious: higher $l$-values have larger frequency differences around the peak difference near $3800 \mu \mathrm{Hz}$. In general, the maximum value of $\Delta \nu / \nu$ was proportional to $B^{2}$, and the frequency of maximum change was a function of both $B^{2}$ (larger fields moved the peak toward lower frequencies) and particularly $M_{D}$ (a deeper perturbation also moved the peak toward lower frequencies). For frequencies less than $2500 \mu \mathrm{Hz}$, the magnitude of $\Delta \nu / \nu$ was related to the depth at which the field distribution penetrated into the solar interior (i.e., was deter- mined by $M_{D}$ and $\sigma$ ). Such sensitivity to the distribution and location of the magnetic field cannot be explained solely in terms of a radius change but reflects structural changes occurring throughout the entire solar model.

The most unusual feature in the calculated changes displayed in Figure 1 was the precipitous "drop" near $5000 \mu \mathrm{Hz}$, very close to the acoustic cutoff frequency but located roughly $500 \mu \mathrm{Hz}$ greater than might be expected from an extrapolation of the observed changes. The variation of such high-frequency modes has been the subject of many recent efforts (Jain \& Roberts 1994; Hindman \& Zweibel 1994; Johnston, Roberts, \& Wright 1995; Jain 1995), whose results can be summarized by stating that the behavior of the high-frequency modes is extremely sensitive to the details of the solar atmosphere. For our solar models, modes between $4800 \mu \mathrm{Hz}$ and $5200 \mu \mathrm{Hz}$ demonstrated relative frequency differences of roughly -0.03 (i.e., far beyond the scale of Fig. 1) before returning to positive changes on order of $10^{-4}$ at frequencies near and beyond 5400 $\mu \mathrm{Hz}$. We do not wish to dwell on the variation of these very high frequency modes and instead defer to the provided references.

\section{SECOND ATTEMPT TO REPRODUCE CHANGES}

After quantifying the effects of location, intensity, and distribution on the $p$-mode changes, the next step was to attempt a better fit to the observations. By skewing the Gaussian distribution toward deeper regions, we were able to match the observations for frequencies less than $3000 \mu \mathrm{Hz}$ and greater than $4000 \mu \mathrm{Hz}$. In fact, the observations were fit ideally not by a Gaussian but by a truncated Gaussian (Fig. 2). 


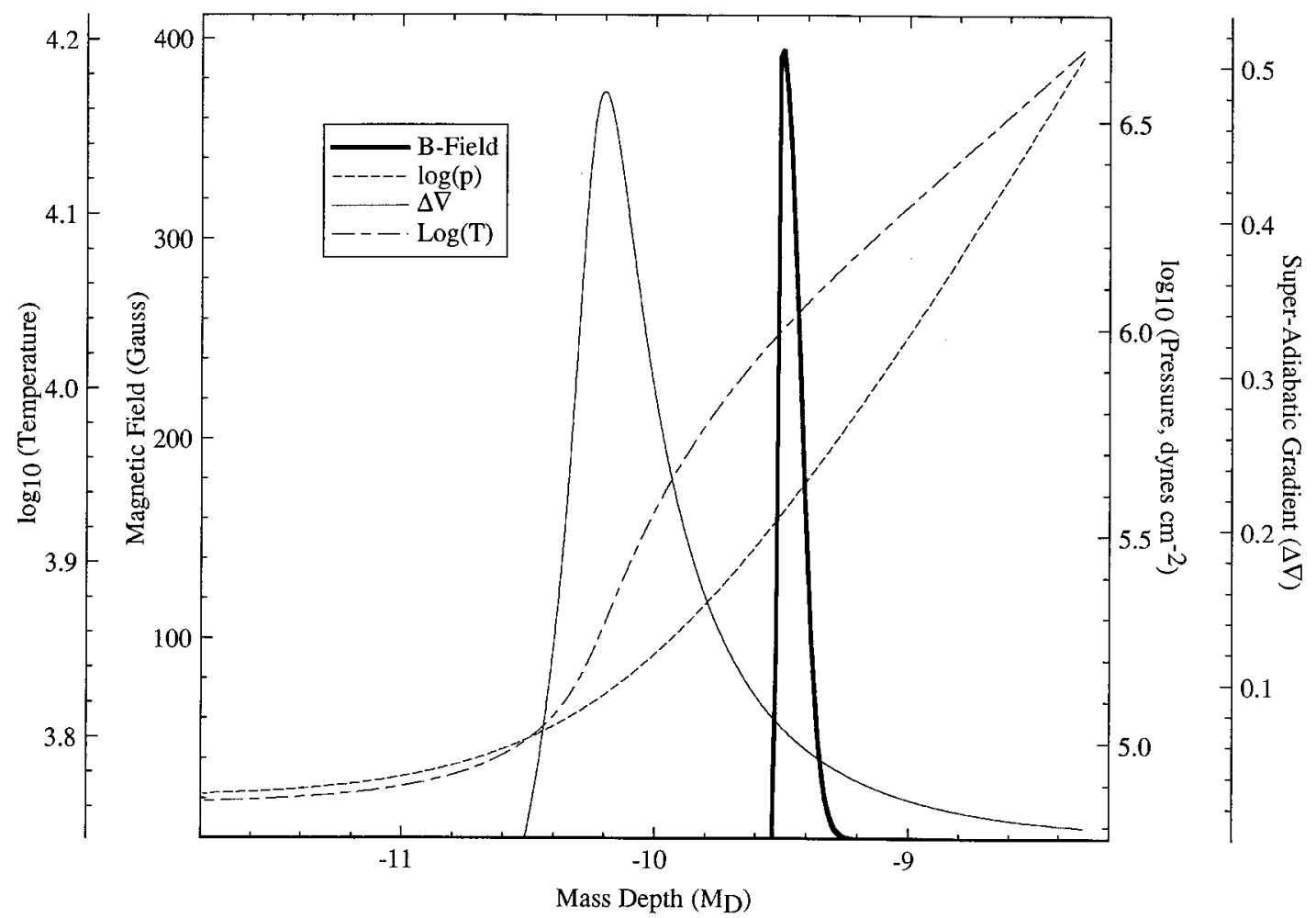

FIG. 2.-The proposed change in the magnetic field distribution and intensity between 1989 and 1986. Also plotted is the variation of the temperature, pressure,

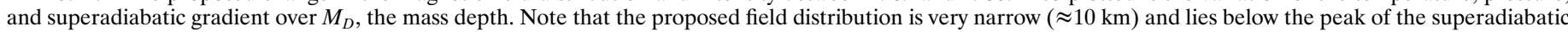
gradient entirely within the convection zone.

Unfortunately, the inclusion of a discontinuity in the magnetic field often made for erratic values of $\Delta \nu / \nu$ which tended to scatter about the observed distribution displayed in Figure 1. We believe that the scatter was a numerical effect which is being addressed by increasing the spatial resolution of future solar models.

\section{SPECULATION}

Assuming that the change in magnetic field distribution described in the previous section was correct, we were faced with an apparent contradiction. Stability analyses have indicated that any magnetic field should be immediately ejected by buoyancy forces from a convective region. In addition, diffusive effects, enhanced by convective motions, should dissipate even a mysteriously stable field over periods much less than $2 \mathrm{yr}$. We suggest that if our results withstand further scrutiny, then the proposed change in the field could be used to argue that magnetic fields may obey a stability criterion which is different from the criterion governing the convection. In other words, a large-scale magnetic field may be dynamically stable throughout part of the solar convection zone. This statement may be supported by the field distribution in Figure 2. Note that no field variation in the region given by $M_{D}<-9.5$ is required to reproduce the observations; buoyancy effects may keep this region free of magnetic field at all times. Between 1986 and 1989, however, changes associated with the solar cycle could have pushed the point of stability deeper within the Sun, slowly clearing out a narrow region of previously stable magnetic field. As indicated by the width of the field distribution, the change in the depth of the hypothetical stability criterion would have been small.
Regardless of the veracity of our spectulation, several sobering scientific results bear mentioning. The structure of the entire convection zone is enormously sensitive to small changes occurring close to the peak of the superadiabatic gradient; a variation of only several kilometers in the location of the proposed stability criterion could result in large and observable variations in the solar radius, luminosity, surface temperature, and $p$-mode frequencies. At the same time, we must acknowledge that between 1986 and 1989 changes in solar structure and/or magnetic field distribution may have occurred elsewhere in the solar interior without yielding observable changes in the $p$-mode frequencies. For example, large-scale magnetic field changes may have occurred near the base of the solar convection zone and would have produced their own "signature" frequency changes. Our research indicates, however, that in order to yield frequency changes such that $\Delta \nu / \nu \approx 10^{-4}, \Delta B \geq 10^{6} \mathrm{G}$ near the convection zone base (resulting in $\Delta L / L \approx \Delta R / R \approx 10^{-5}$ at the solar surface). If changes on the order of $10^{5} \mathrm{G}$ occurred, the effect on the $p$-modes would have been undetectable in the data presented in Figure 1. Between 1986 and 1989, observed changes in magnetic field intensity occurred near the solar surface. Our research indicates, however, that any field change in this region yielding $\Delta \nu / \nu \approx 10^{-4}$ also produced $\Delta L / L \approx 10^{-2}$; conversely, field changes at the surface producing more reasonable luminosity changes do not yield observable changes in the $p$-modes. In summary, while we cannot argue against magnetic field changes elsewhere in the Sun between 1986 and 1989, we can say such changes must have had a much smaller effect on the $p$-mode frequencies than the magnetic field changes we are advocating near $M_{D}=-9.5$. 


\section{RADIUS AND LUMINOSITY VARIATIONS}

Our proposed magnetic field variation also made for changes in the global properties of the solar models. The proposed decrease in field intensity between 1986 and 1989 was accompanied by a decrease in the solar radius of approximately $4.2 \mathrm{~km}$ and an increase in the solar luminosity of roughly $0.25 \%(\Delta \log R / \Delta \log L \approx-0.0024$; the luminosity increase corresponded to an increase in the effective temperature of roughly $3.6 \mathrm{~K}$ ). While an increase in the solar luminosity of $0.1 \%$ may have occurred between 1986 and 1988 (Willson \& Hudson 1988), the difficulties of distinguishing irradiance variations from luminosity variations may mitigate the discrepancy between theory and observation. Radius changes as small as several kilometers are detectable only by the Solar Disk Sextant Experiment (SDS; Sofia et al. 1984; Sofia, Heaps, \& Twigg 1994a; Sofia et al. 1994b), which measured a solar radius increase of roughly $4 \mathrm{~km}$ between 1992 and 1994 (Sofia, Heaps, \& Twigg 1995). If the observed change in the $p$-modes was the result of changes associated with the solar cycle, then, for the periods of 1986-1989 and 1992-1994, one might expect a radius change of similar magnitude (since the same phenomena was operating at both times) but opposite sign (since the two periods are on opposite sides of a solar maximum). Such a picture is consistent with the available SDS data.

\section{SUMMARY}

We contend that the observed $p$-mode changes of Libbrecht \& Woodard (1990) can be explained in terms of a sharp drop in the magnetic field intensity within a narrow region near the top of the solar convection zone between 1986 and 1989. According to our models, the observed change in the $p$-mode frequencies was accompanied by a decrease in the solar radius of approximately $4 \mathrm{~km}$ and an increase in the solar luminosity of roughly $0.25 \%$. Such changes are consistent with available observations, but more data are needed to reveal the mechanism linking the frequencies changes, the radius changes, the luminosity changes, and the solar cycle.

This research was supported in part by an appointment to the Global Change distinguished Postdoctoral Fellowships sponsored by the US Department of Energy, Office of Health and Environmental Research, and administered by the Oak Ridge Institute for Science and Education. D. B. G. acknowledges the support of NSERC.

\section{REFERENCES}

Endal, A. S., Sofia, S., \& Twigg, L. W. 1985, ApJ, 290, 748

Goldreich, P., Murray, N., Willette, G., \& Kumar, P. 1991, ApJ, 370, 752 Guenther, D. B. 1994, ApJ, 422, 400

Guenther, D. B., Demarque, P., Kim, Y.-C., \& Pinsonneault, M. H. 1992, ApJ, 387,372

Hindman, B. W., \& Zweibel, E. G. 1994, ApJ, 436, 929

Jain, R. 1995, in Proc. 4th SOHO Workshop, Helioseismology, in press Jain, R., \& Roberts, B. 1993, ApJ, 414, 898

1994, A\&A, 286, 254

Johnston, A., Roberts, B. \& Wright, A. N. 1995, in ASP Conf Ser. Vol. 76 GONG '94: Helio- and Astro-Seismology, ed. R. K. Ulrich, E. J. Rhodes, Jr., \& W. Däppen (San Francisco: ASP), 264
Kuhn, J. R. 1995, in Proc. 4th SOHO Workshop, Helioseismology, in press Libbrecht, K., \& Woodard, M. 1990, Nature, 345, 779 Lin, H. 1995, ApJ, 446, 421

Lydon, T. J., \& Sofia, S. 1995, ApJS, 101, 357

Sofia, S., Chiu, H.-Y., Maier, E., Schatten, K. H., Minott, P., \& Endal, A. S. 1984, Appl. Opt., 23, 1235

Sofia, S., Heaps, W., \& Twigg, L. W. 1994a, ApJ, 427, 1048

. 1995, ApJ, submitted

Sofia, S., Heaps, W., Twigg, L. W., Lydon, T. J., \& Sofia, U. J. 1994b, BAAS, 26, 1377

Willson, R. C., \& Hudson, H. S. 1988, Nature, 332, 810 